\title{
Viscosity model of deep eutectic solvents from group contribution method
}

\author{
Liuying $\mathrm{Yu}^{1}$, Xiaojing $\mathrm{Hou}^{2}$, Gao-Peng Ren ${ }^{1}$, Kejun $\mathrm{Wu}^{3}$, and Chao-Hong $\mathrm{He}^{4}$ \\ ${ }^{1}$ Affiliation not available \\ ${ }^{2}$ Zhejiang University \\ ${ }^{3}$ University of Leeds \\ ${ }^{4}$ State Key Laboratory of Chemical Engineering
}

January 11, 2022

\begin{abstract}
In this work, based on mathematical model inspired by transition state theory, the group contribution (GC) method is used to predict the viscosity of DESs. The model is constrained by Eyring rate theory and hard sphere free volume theory. A dataset of 2229 experimental measurements of the viscosity of 183 DESs from literature is used for determining the model parameters and subsequent verification of the model. The rules introduced by this model are simple and easy to understand. The results show that the proposed model is able to predict the DESs viscosity with very high accuracy, i.e., with an average absolute relative deviation of $8.12 \%$ over the training set and $8.64 \%$ over the test set, using only temperature and composition as inputs. The maximum absolute relative deviation is $34.63 \%$. Therefore, the as-proposed model can be considered a highly reliable tool for predicting DESs viscosity when experimental data are absent.
\end{abstract}

\section{Hosted file}

Viscosity model of deep eutectic solvents from group contribution method.docx available at https://authorea.com/users/454887/articles/552363-viscosity-model-of-deep-eutecticsolvents-from-group-contribution-method 